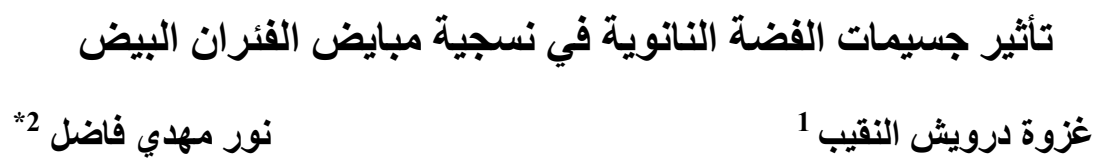

\author{
استلام البحث 2017/28 \\ قبول النشر2017/5/24
}

This work is licensed under a Creative Commons Attribution 4.0 International License.

تهدف الدر اسة إلى التعرف على التغير ات النسجية لمبيض الفئران البيض Albino Mice المتعرضة لجسيمات الفضة النانوية جمعت 60 انثى بالغة ،جر عت بجسيمات الفضة النانوية Silver Nano Particles (SNP)

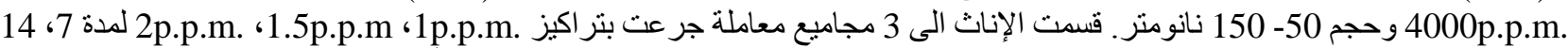

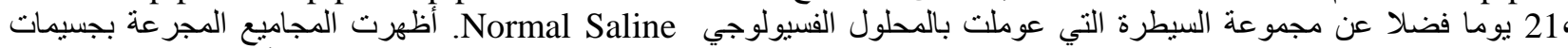

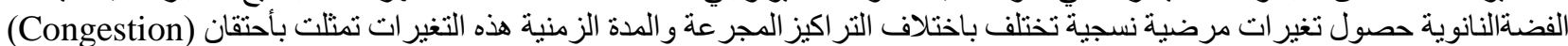

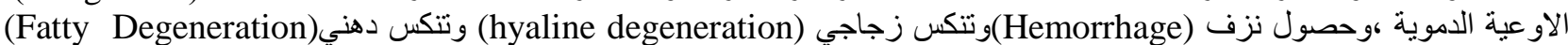

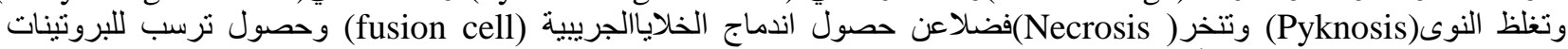
المتمثلة بالو Amyloid داخل الأوعية الدموية وحصول تنخردهني ( Fytty necrosis) .

الكلمات المفتاحية: جسيمات الفضة النانوية ، المبيض ، تأثير ات مرضية نسجية.

المواد وطرائق العمل:

جمع الحيوانات واقلمتها

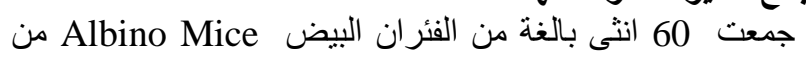

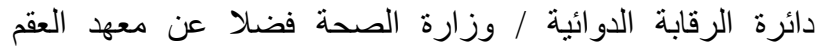

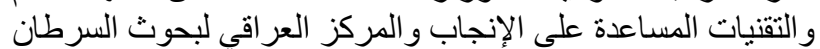

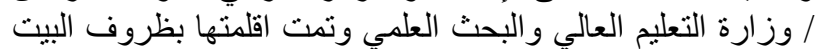

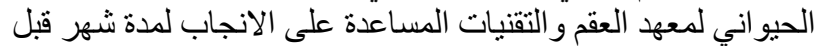

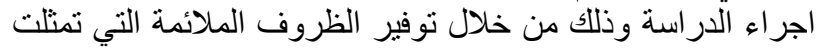

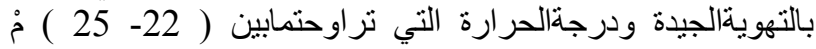

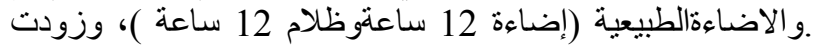

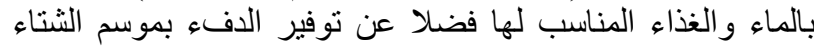

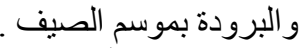
مجاميع المعاملة والسيطرة

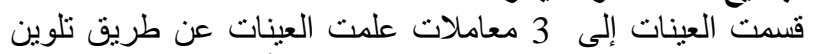

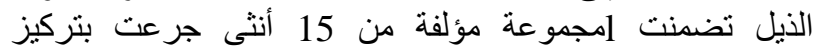

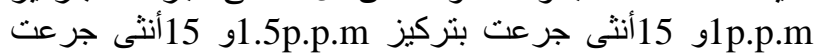
بتركيز 2p.p.m من محلول جسيمات الفضة النانوية (SNP) لمدة

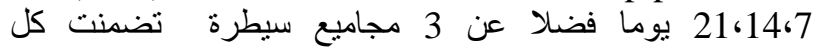

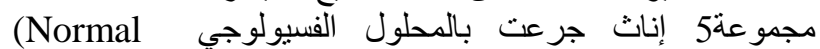
m حقن داخل البريتون Saline) (Intra peritonial injection)

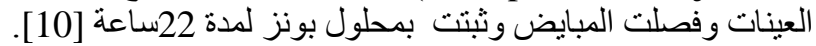
التحضيرات النسجية

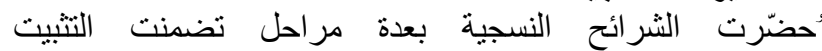
(Fixation) الترويق (Clearing) و الارنشاح والطمر (Fixfiltration and

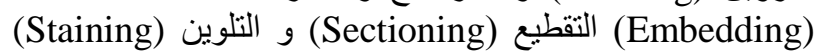
[10] الفحص و التصوير المجهري (Microscope examination) .and photography)
تتمثل الجسيمات النانوية Nanoparticles منعناقيد (clusters) من الذرات (atoms) والتي يتراوح اني معدل حجمها مابين(1)

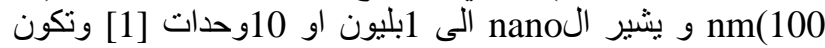

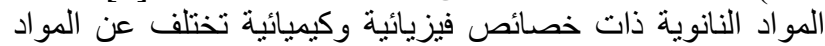

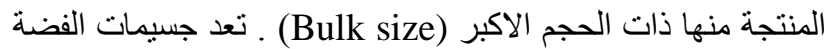

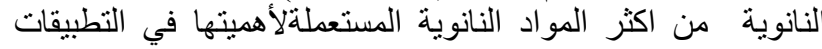
الطبية والبايولوجية (Medical \&Biological Application) (Anti- inflammatory لتميزها بخصائص مضادة للالتهاب (An) (Anti- Viral Properties) و والفايروسات Properties)

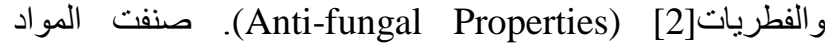

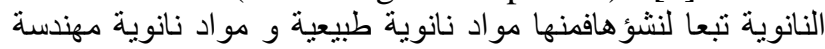

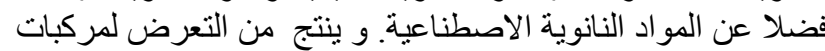

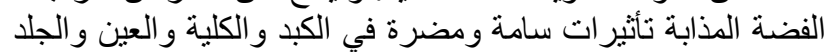

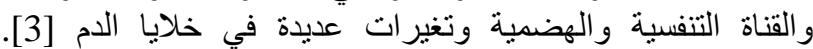

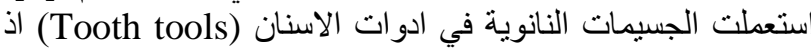

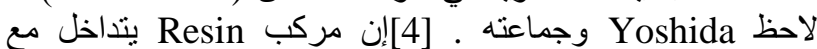

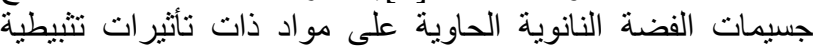

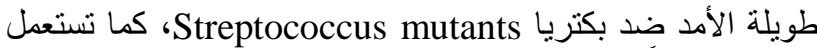
بوصفها مواداً علاجية في علاج العديد من الأمراض كالسرطان

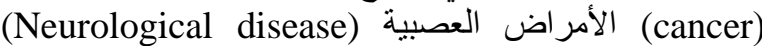
[5]والعدسات اللاصقة للعين[6] كما تستعمل بوصفها مضادات

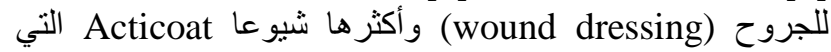

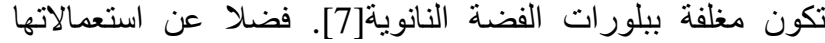
المفيدة فانه توجد تأثير ات نسجية مرضية لجنية لجسيمات الفضة النية النانوية توصل [8] الى حدوث التهاب بكبد الجرذان مع توسع للجيبانيات

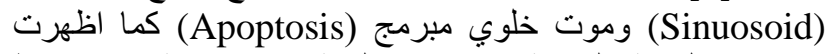

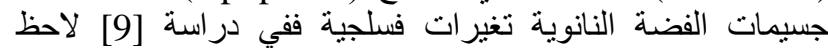

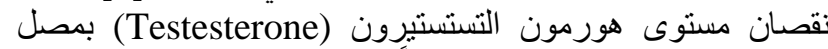
الذكور البالغة المجر عة مدة 28 يوماً وبتر اكيز مختلفة الفئ.

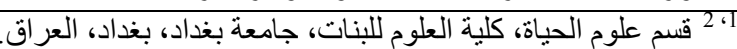

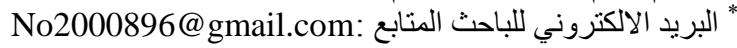


لنوى(Pyknosis) خلايا السدى مع تتخر(Necrosis) في خلاياه فضلا عن حصول اندماج الخلايا (fusion cell) الخلايا الجريبية

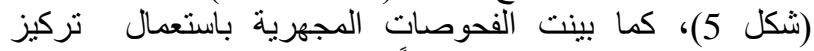

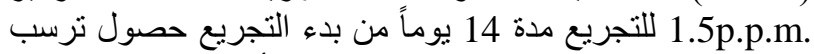
للبروتينات المتمثلة بال(Amyloid) داخل الأوعية الدموية ( شكل

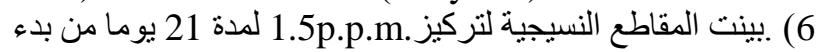
التجريع ظهور التنخر بخلايا السدى وتتكس زجاجي في في خلايا

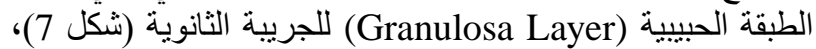

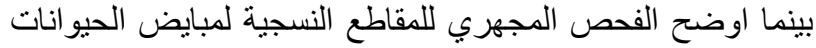

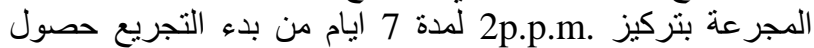
تتكس زجاجي في خلايا السدى والخلايا الجريبية للطبقة الحبييية في

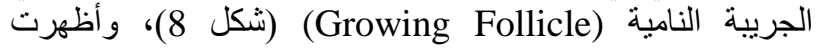

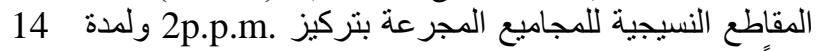
يوماًاستمر ار التتكس الدهني و التنخر الدهني (Fatty Necrosis)

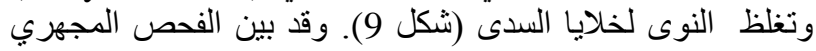

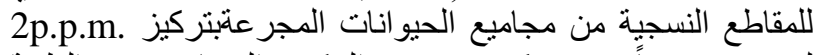

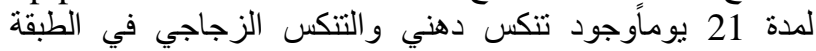
الحبيبية للجريب الثانوي. (شكل 10 10).
النتائج :

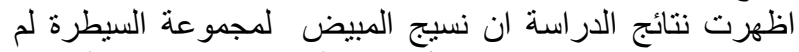
يظهر تغيير اتنسجية كما في(شكل 1) ، لوحة الوحظ عند فحص الفص المقاطع

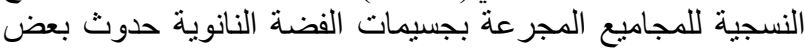

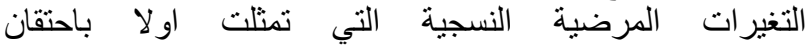

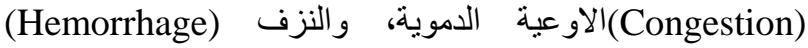

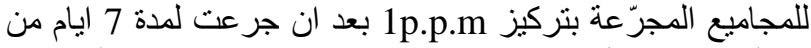

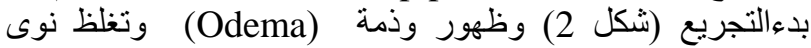
(Pyknosis) البيضة(Oocyte) فضلا عن تتخر الخلية البيضية بالجريبة الثانوية

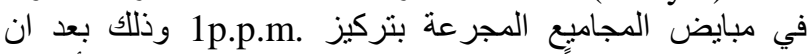

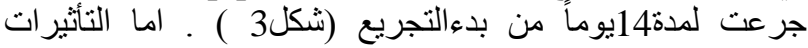

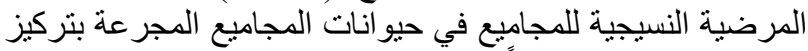
1p.p.m.

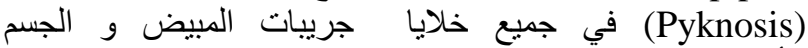
الأصفر(شكل 4).بينت المقاطع النسجية للمجاميع المجّرعة بتريات

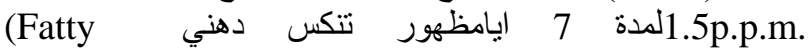
degeneration)

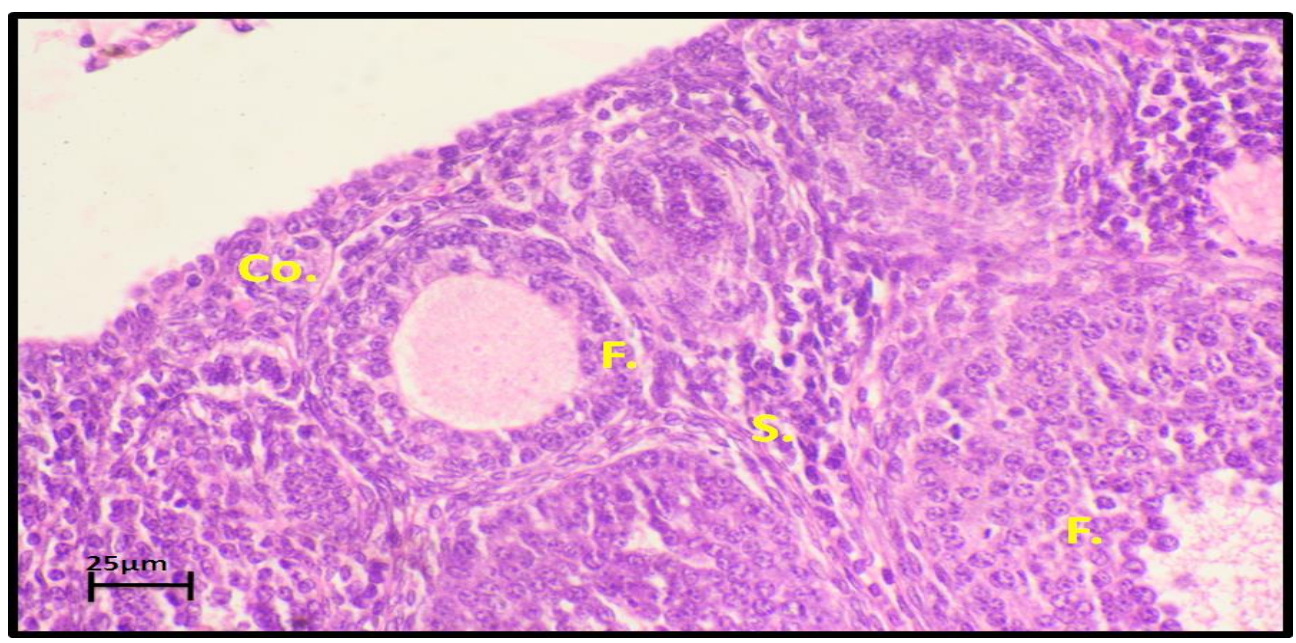

شكل 1. مقطع مستعرض في مبيض انثى عذراء من مجموعة السيطرة(Control) يوضح منطقة القشرة (Cortex (Co.) Coma) السدى (4trol) (400X) (H\&E) .Follicles (F.) Stroma (S.)

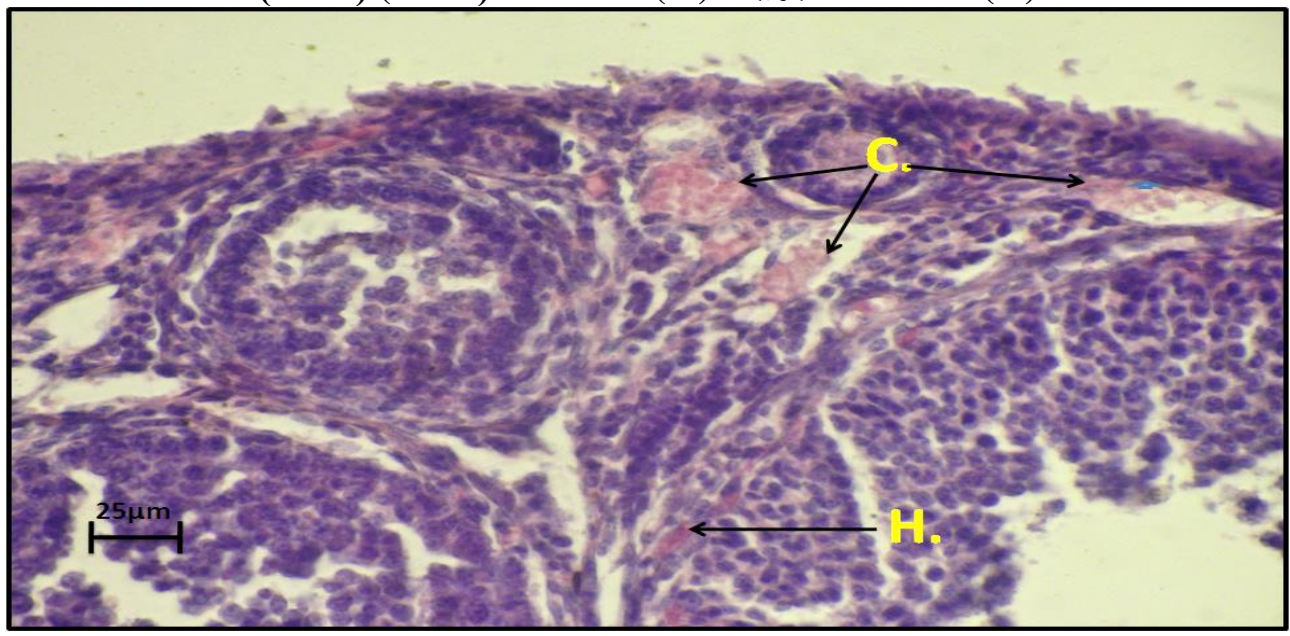

شكل 2. مقطع مستعرض في مبيض انثى عذراعمجرعة بجسيمات الفضة النانوية)(SNP.p.m.تركدة 7ايام يوضح احتقان

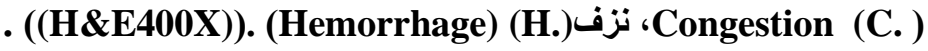




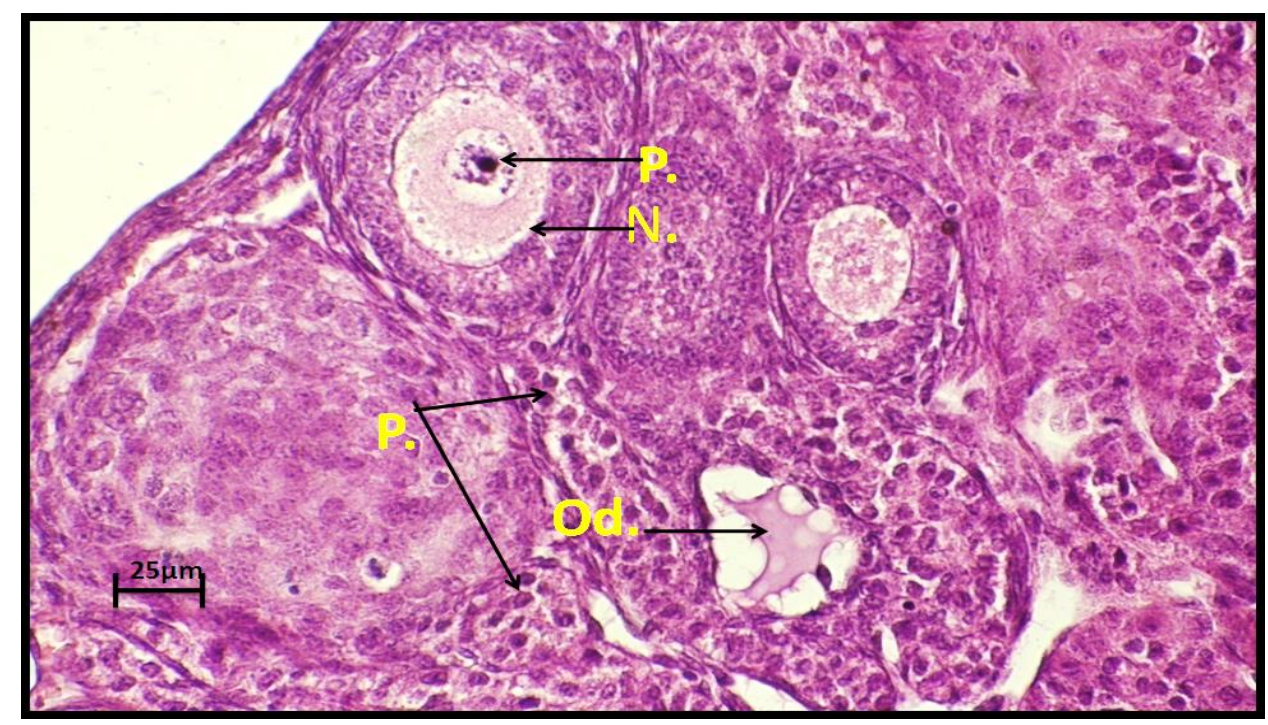

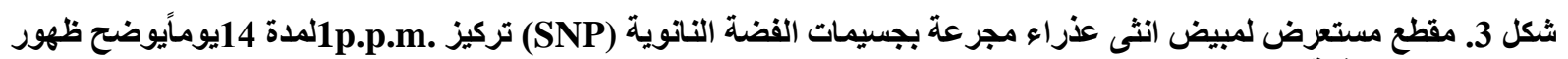

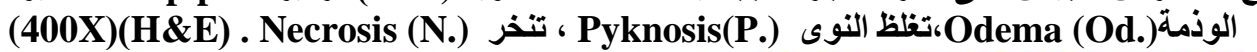

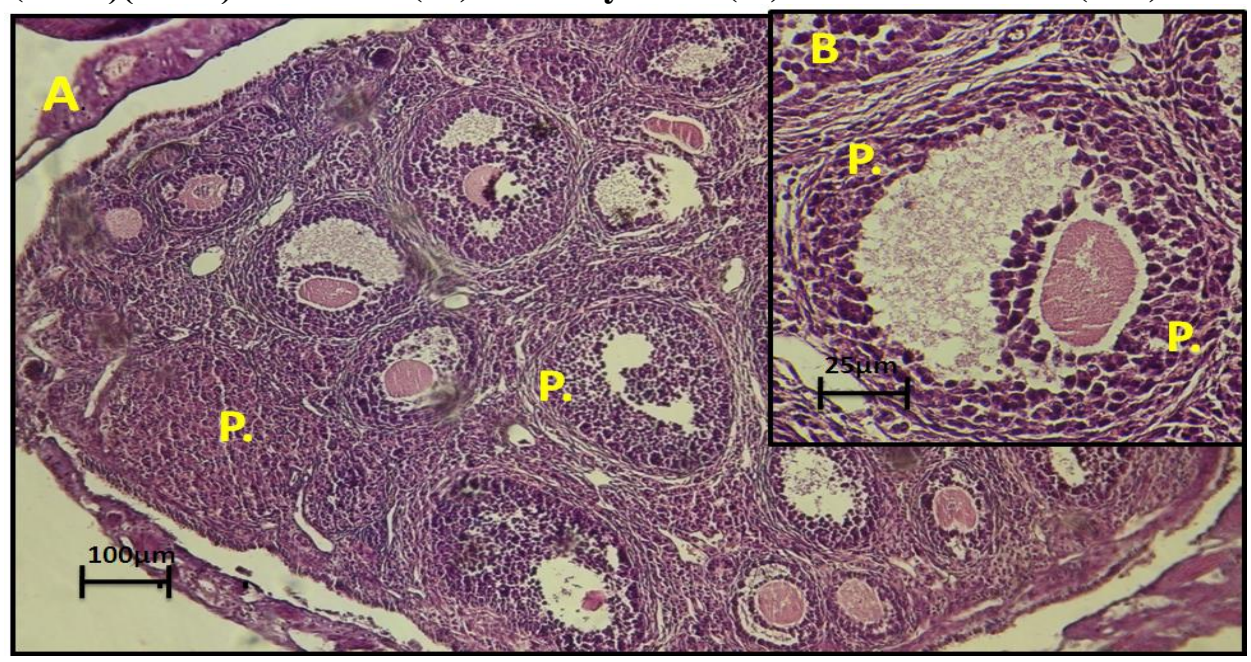

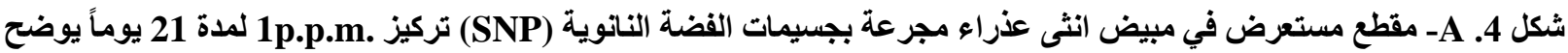

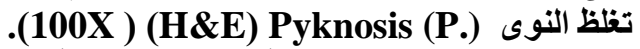

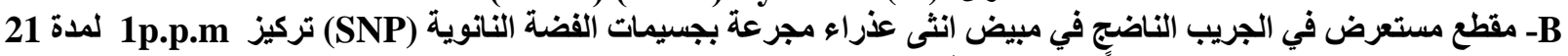
يوماً يوضح تظلظ النوى (400X)(H\&E).Pyknosis (P.).

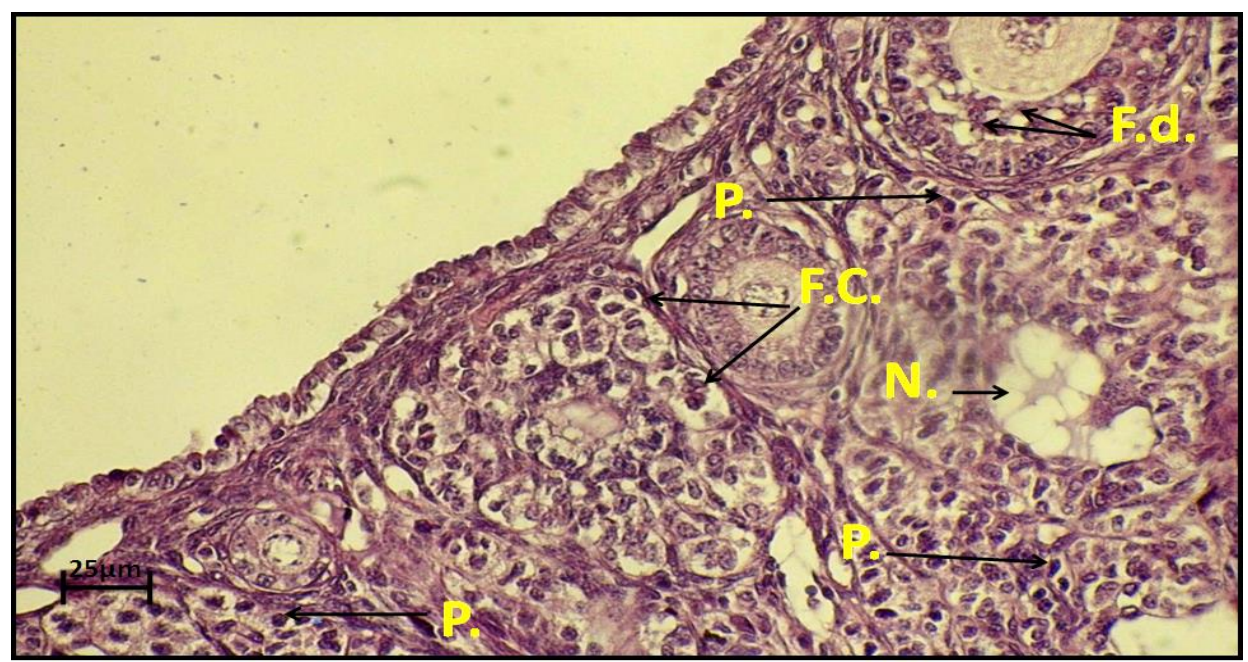

شكل 5. مقطع مستعرض في مبيض انثى عذراء مجرعة بجسيمات الفضة النانوية (SNP) تركيز 1.5 p.p.m. تبد ايام يوضح اندماج

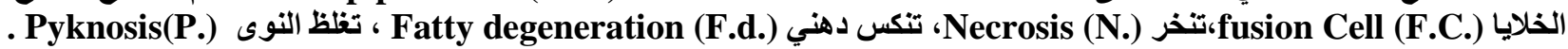
$(400 X)(H \& E)$ 


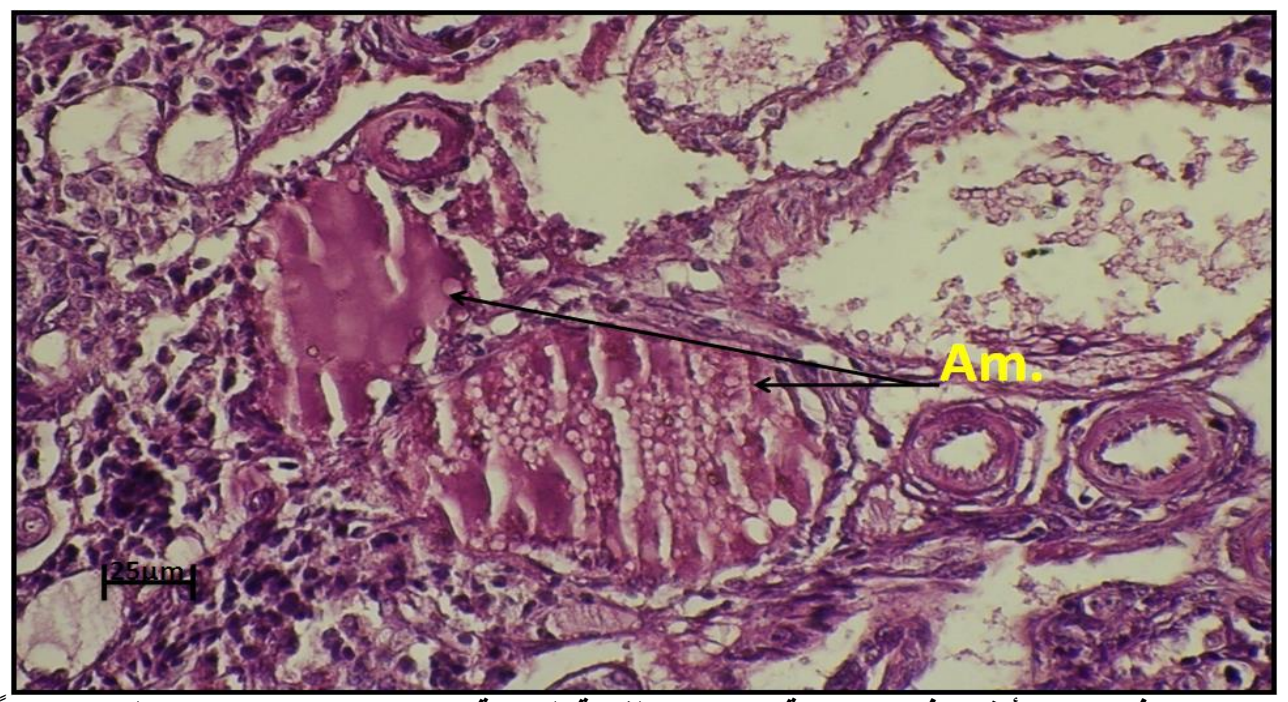

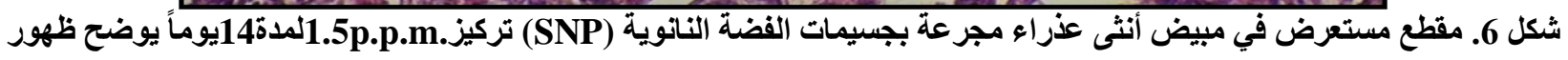

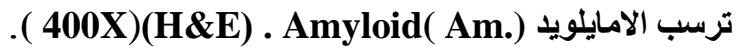

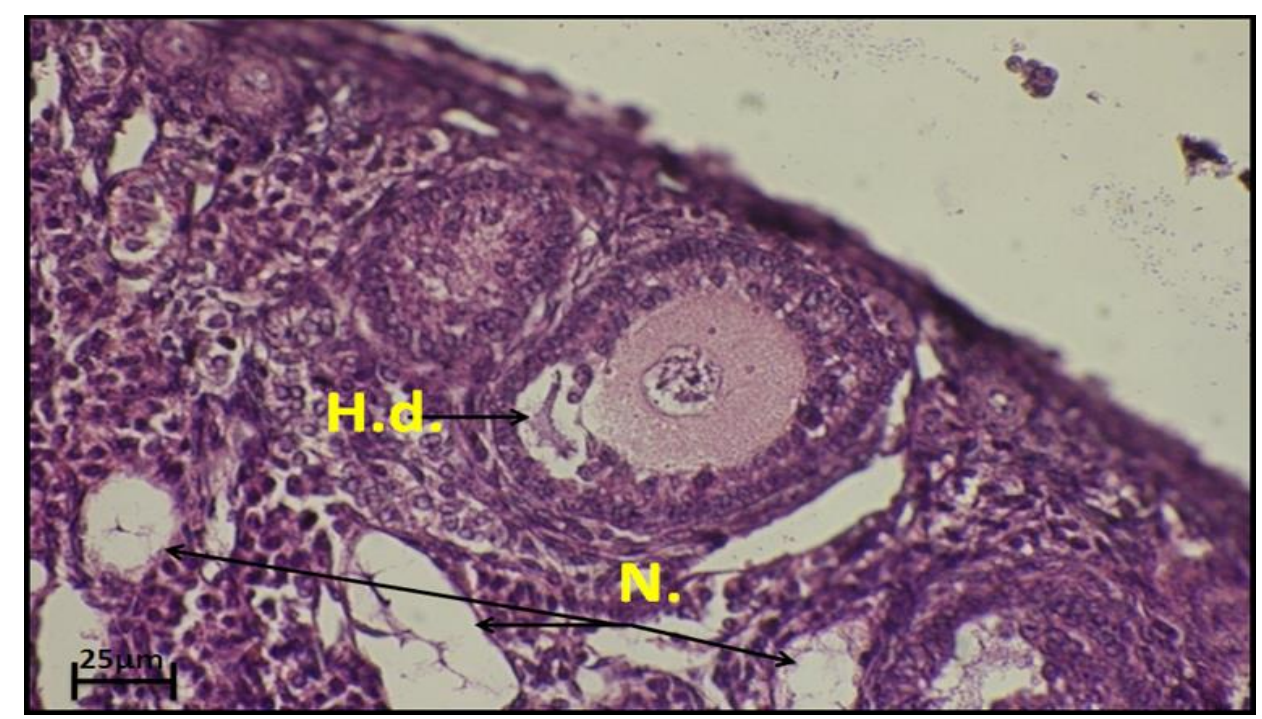

شكل 7. مقطع مستعرض في مبيض انثى عذراء مجرعة بجسيمات الفضة النانوية 211.5 p.p.m تمدة 21يوماً يوضح تنخر (400X) (H\&E) Hyaline degeneration (H.d.) تنكس زجاجي (Necrosis (N.)

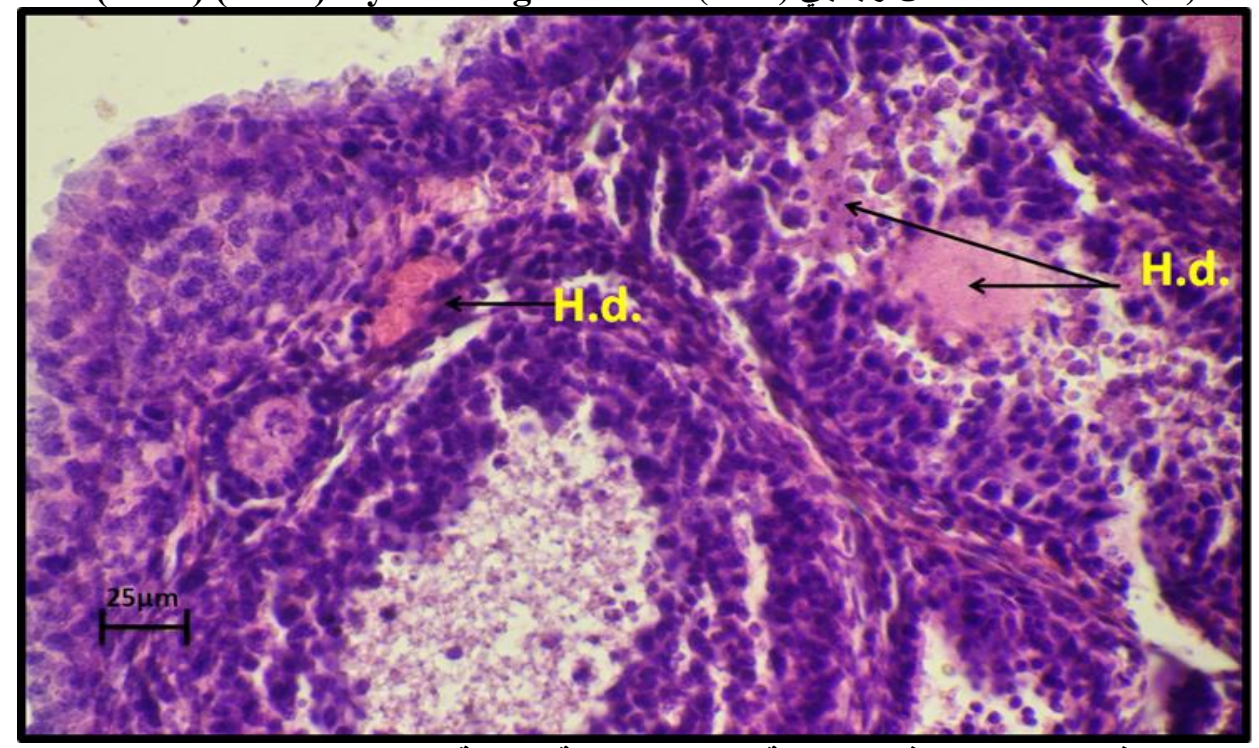

2p.p.m. لمدة 7ايام يوضحتنكس

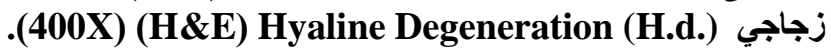




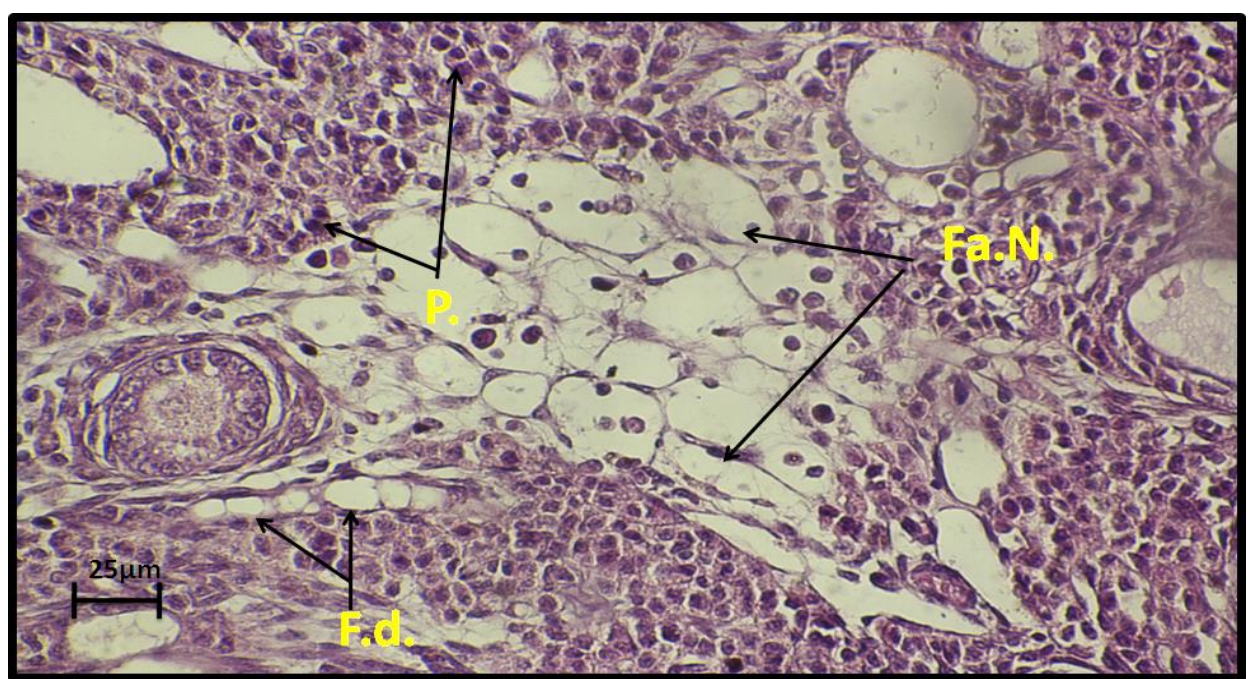

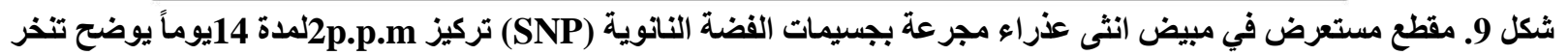

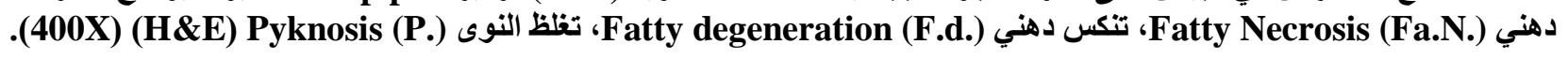

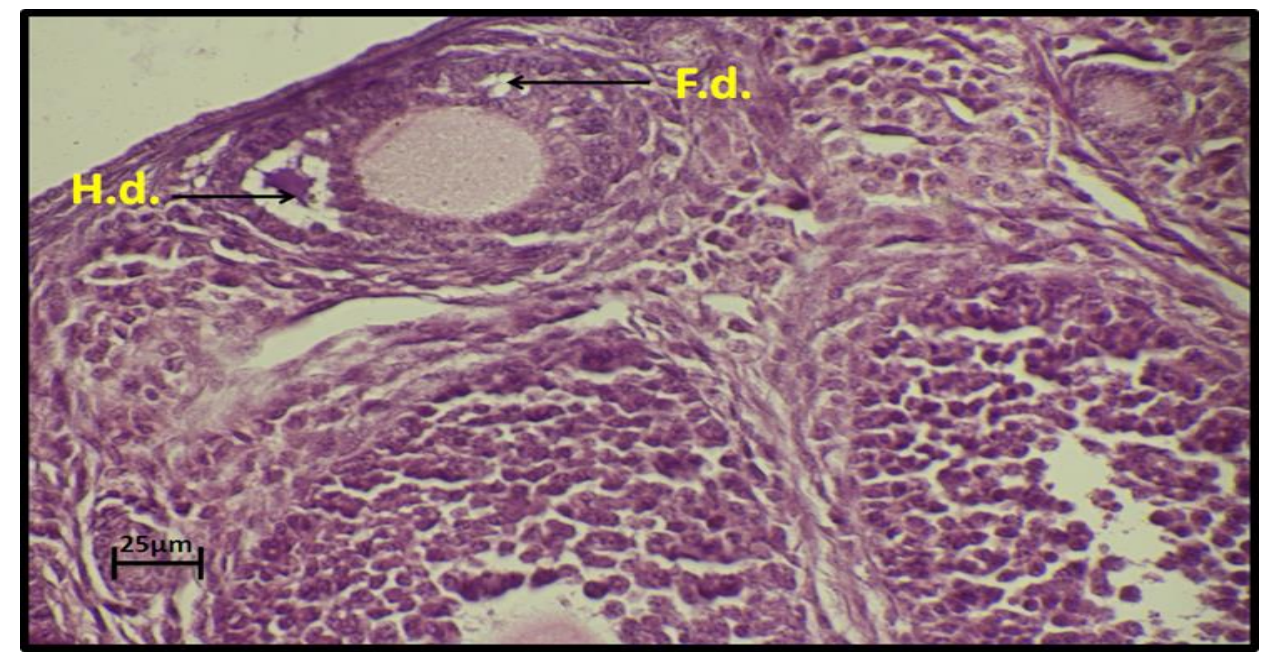

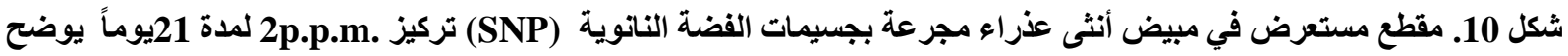

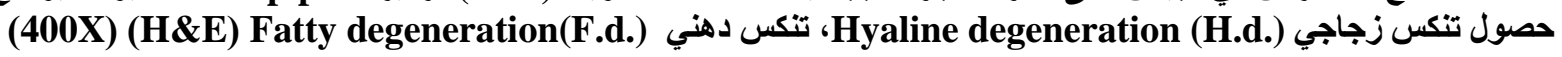

البرينون (Intraperitoneal Injection) للجرذان الى حدوث

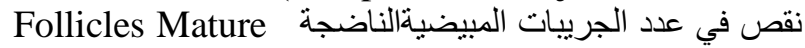
وذلك لاخول الفضة النانوية النخلايا المبيض ممائية النائدي الى حالة

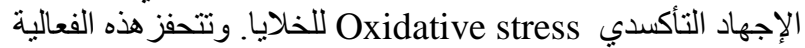

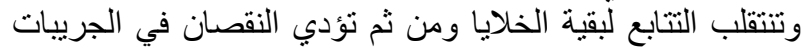

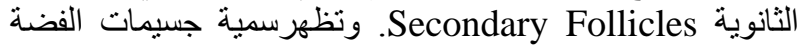
النانوية بالدرجة الاساسية من خلال تنشيطها لعطلية الإجهاد

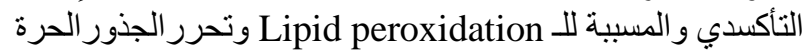

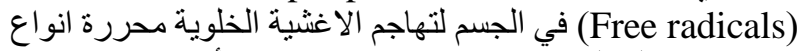

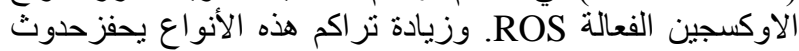

الاستجابة الالتهابية [11] الفية. وتتثنل اولى التأثيرات السلبية الناجمة عن الاستجابات الالتهابية

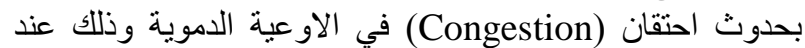

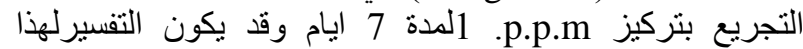
التغييرالنسجي هوتأثير الجسيمات النانوية في جدران الأونية

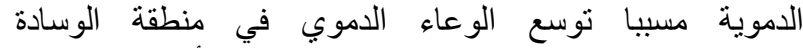
(Microcirculation)

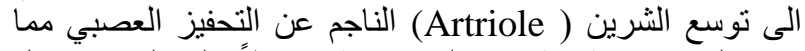

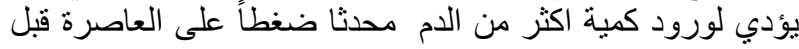
الثعبرية (Precapillary sphincter) وتر اكم الدم في منطقة لئمة
المناقثة :

تسبب جسيمات الفضة النانوية تأثير ات سمية جانبية في الأنسان

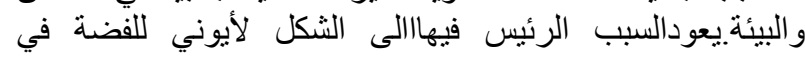

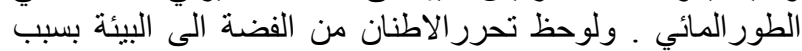

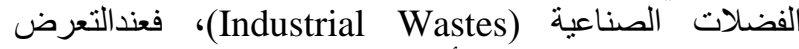
لمركبات الفضة الذائبة ينتج تأثير ات سمية في اعضاء جسم الانسان

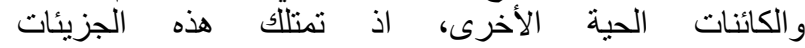
ومنها جزيئات الفضة النانوية نأثيرات منباينة الفئة (Nanoparticles)

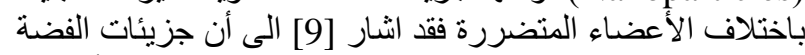

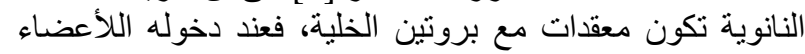

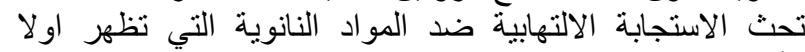

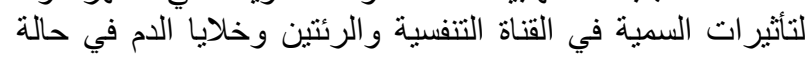

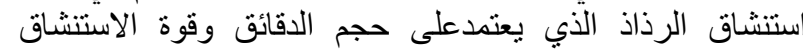

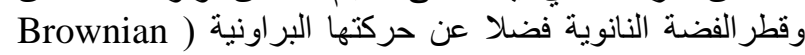

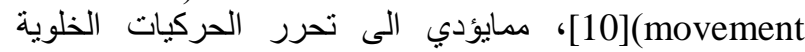
ودخول جسيمات الفضة النانوية الى جهاز الدوران (Cytokines)

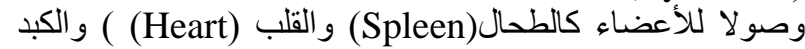

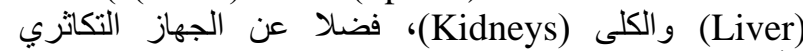

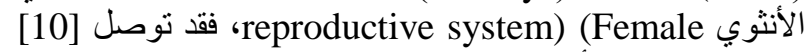
في دراسته لتأثير الفضة النانوية عند الحقن داخل تُصل 
يؤدي الى الخلل في عمليات انتقال المواد بين داخل الخلية (Intracellular) بسبب حدوث خلل بتصنيع البروتين نتيجة تاثير ايونات الفضة

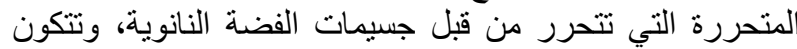

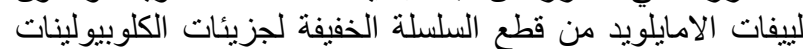
المناعية وربمايحدث الامايلويد نتيجة بلعمة الكيلة الكلوبيولينات الينات المناعية

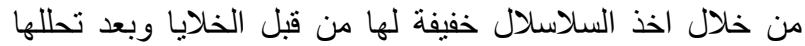

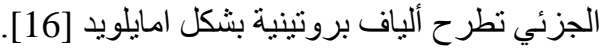

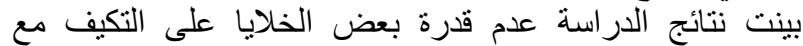

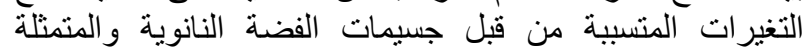

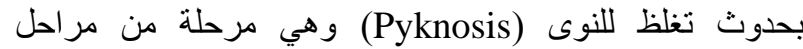

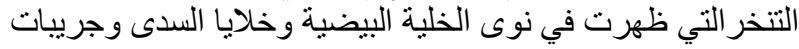

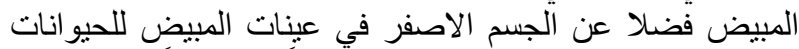

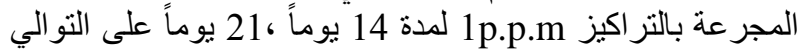

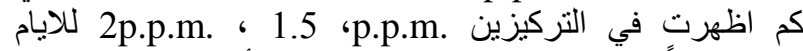
79 14 يومأعلى التو الي متمثلة بتنخر الخلايا بتأثير العو امل المحفزة

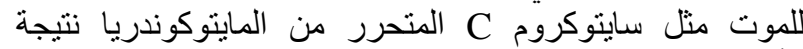

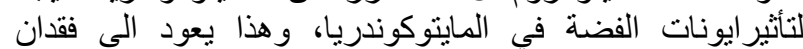

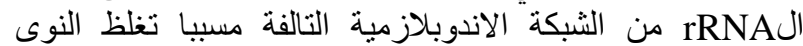

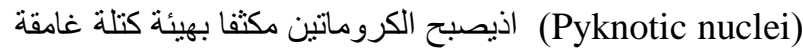

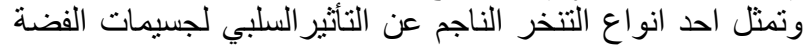

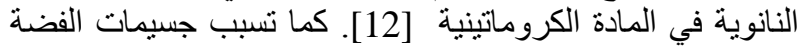

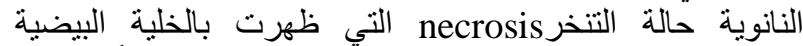

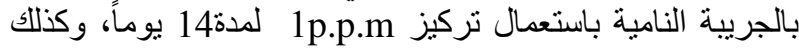

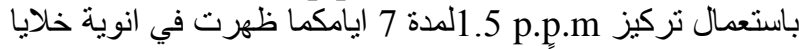

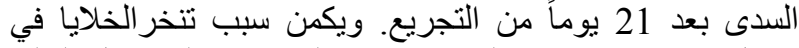

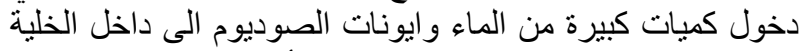

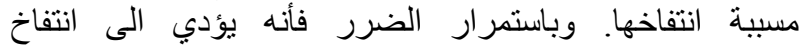

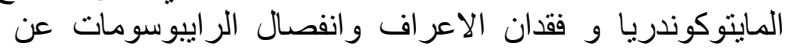

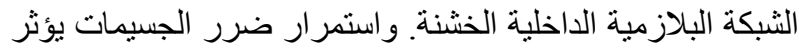

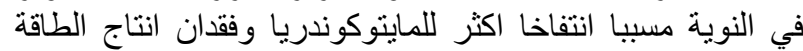

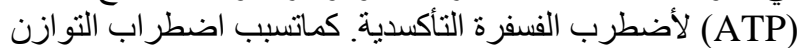

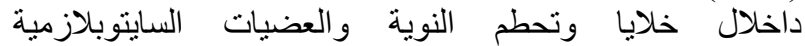

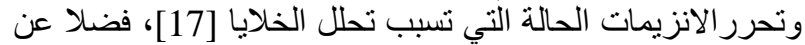

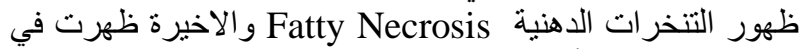

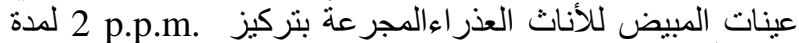

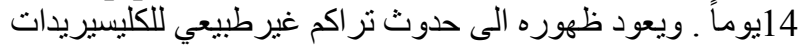

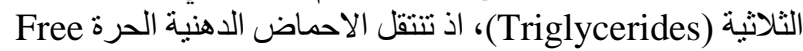
(fatty acids) الأسترةالى كليسيريدات ثلاثية و اخير ا كولسترول (Cholesterol)

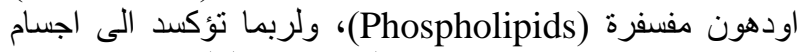

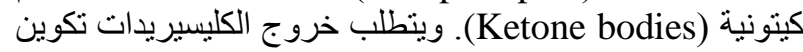

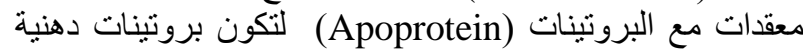

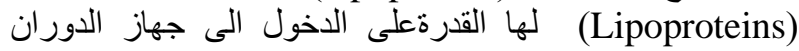

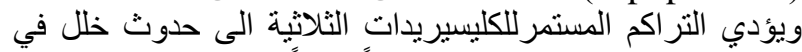

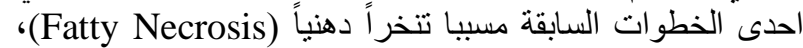

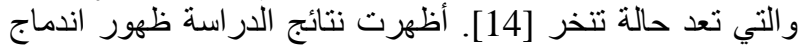
الخلايا fusion Cell التي ظهرت بخلايا السدى بعينات المبيض

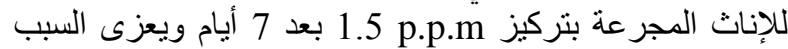

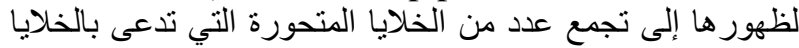

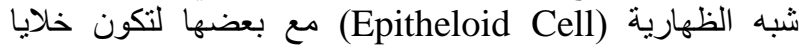
عملاقة متعددة الانوية تثترك بالسايتوبة الفازم نفسه نتيجة الالتهاب

المزمن الحاصل بفعل جسيمات الفضة النانوية [18].
الوسادة الثعبرية (Capillary bed) مؤديا الى جريان دموي

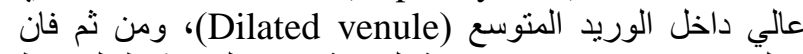
عمليتي توسع واسترخاء الاوعية الدموية تؤدي التى تنشيط الوسائط

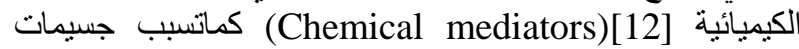
الفضة النانوية حالة النزف الدموي (Hemorrhage) التي ظهرت

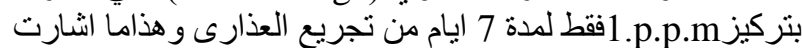

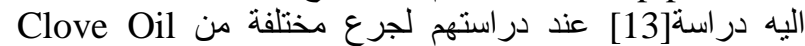

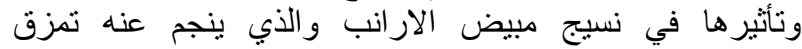
(Rupture)

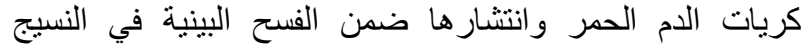

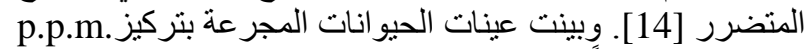

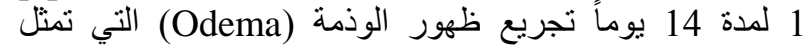

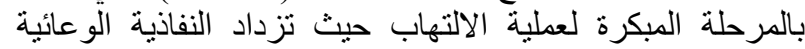
(Vascular permeability) وزيادة حجم الدم المتدفق مؤدية الى زيادة الضغئ (Arterioe)

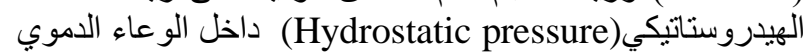

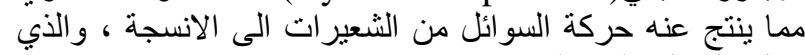

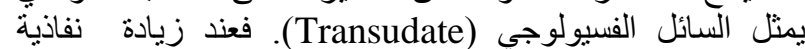
الو عاء الدموي فانه بسمح بحركة السو ائل الغنبة بالبروتين و الخلايا

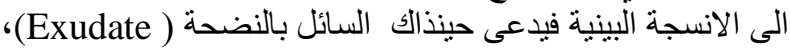

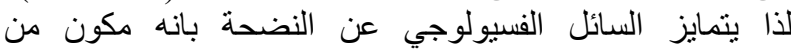

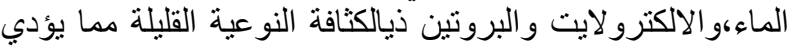

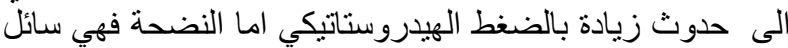

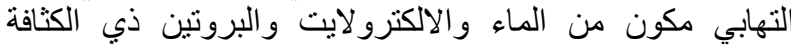

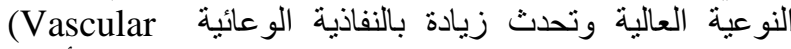
وعermeability)

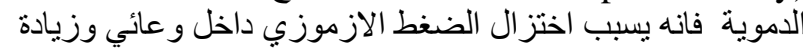

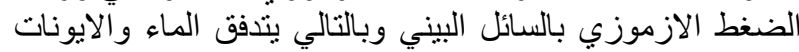

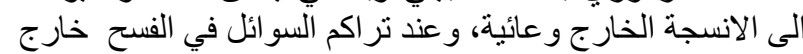

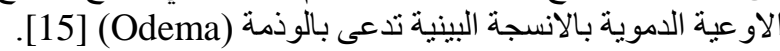
كما بينت الدراسة حالة التنكس الدهني (Fattydegeneration) عنداستعمال التركيز 2 p.p.m ، 2 في تجريع الاناث

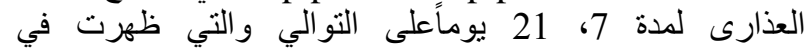

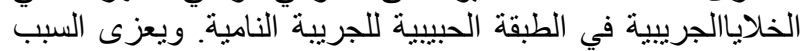
الرئيس للتنكس الدهني لحدوث اضطر ابل بأيض الأحماض الدهنية

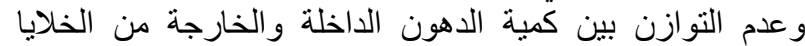

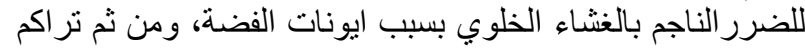
الدهون داخل الخلايا وحصول تفجي (Vaculated) للخلايا التي تلني

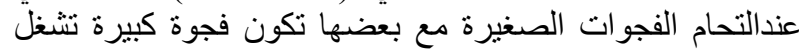

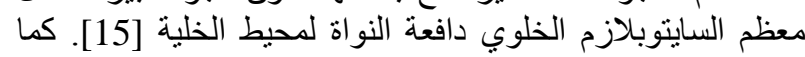
يحدث تر اكم غبرطييحي للكليسيريدات الثلاثية (Triglycerides) اذ تنتقل الاحماض الدهنية الحرة)(Free fatty acids) الى الخلايايا وتتحول بصورة طبيعية بطريقةالأسترة الى كليسيريدات ثلاثية الخية

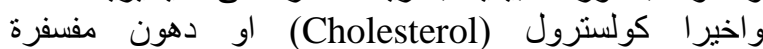
(Ketone ولربما تؤكسد الى اجسام كينونية (Chospholipids)

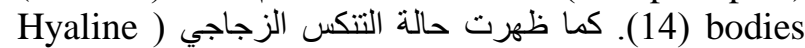
degeneration

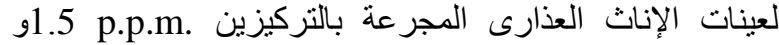
2p.p.m.

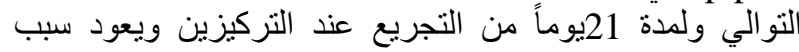

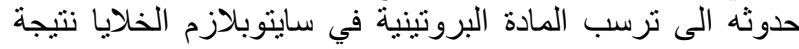
للضرر الذي يحدث في الخلايا الناتج عن جسيمات الفضئة النانوية نتية

اظهرت نتائج الدراسة نرسب الامايلويد (Amyloid) الذئي

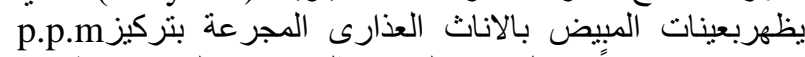
1.5 يترسب في جدران الأوعية الدموية وان تراكم بروتين الامايلويد برئي 
studies. Res. J. of Pharmac. Biol. and chem. 5(4): 1558-1589.

[10]Bancroft, J. D. and Steven, A. S. 2012. Theory and practice of histological techniques. $2^{\text {nd }}$ ed. Churchill living stone, Edinburgh, London: 233-250.

[11]Ghorbanzadeh, V.; Moshtaghian, S. J.; Habibian, S.; Ebadi, A. J. and Vandechali, O. B. 2012. Influence of nanosilver on secondary follicles of ovary via intraperitoneal injection in rats. Euro. J. of Exper. Biol. 2 (4): 1367-1369

[12]Gavanji, S.; Sayedipour, S. S.; Doostmhammdi, M. and Larki, B. 2014. The effect of different concentration of silver nanoparticles on enzyme activity and liver tissue of adult male wistar rats in vivo condition .I.I.S.R.K. 4(2): 182-188.

[13]Dayani, M.; Fathpour, H. and Naghsh, N. 2014. The effect of silver nanoparticles and thioacetamide on blood urea nitrogen and creatinine in male laboratory mice .I. I. B. 4(1): 139-142.

[14]Robbins, S. L.; and Kumar, V. 1987. Basic Pathology. $4^{\text {th }}$ ed Saunders Company, Phildelphia, London: 787.

[15]Kumar, V.; Abbas, A. K.; Fausto, N. and Mitchell, R. N. 2007. Robbins Basic Pathology. $8^{\text {th }}$ ed. John F. Kennedy Blvd. Philadelphia, USA: 85-86.

[16] Al zahid, A. A.; Faris, K. J. and Kodiar, O. S. 2015. Histopathological study of the toxic doses of Clove Oil Syzygiumaromaticum on ovaries of female rabbits. IOSR-JAVS. 8(8): 33-38.

[17]Bhattacharya, G. K. 2016. Concise pathology for exam preparation. 3rd Elsevier. RELX India: 14-15.

[18]Sundriter, W. and Thomas, C. 1979. Color Atlas and Textbook of Histopathology. 6th ed. Sandritter, Walter. USA. : 225.

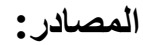

[1] Abou El -Nour, K. M. M.; Eftaiha, A. and AlWarthan, A. 2010. Synthesis and applications of Silver nanoparticles .Ar .J. of Chem. 3(3):135-140.

[2] Zhong, W.; Xing, M. M. and Maibach, H. I. 2010. Nanofibrous materials for wound care. Cutan. Ocul. Toxicol. 29(3):143- 152.

[3] Panyala, NP.; Pena-Mendez, EM. and Havel, J. 2008. Silver or silver nanoparticles ahazardous threat to the environment and human health .J. Appl. Biomed. 6: 117 -129.

[4] Yoshida, K.; Tangagawa, M.; Matsumoto, S.; Yamada,T. and Atsuta, M. 1999. Antibacterial activity of resin composites with silver - containing materials. Eur. J. oral sci. 107(4) :290 -296.

[5] Donner, A. 2010. Nanotechnology in molecular medicine trends in molecular medicine. ESof. 16(12):551 -552.

[6] Tien, DG.; Lioa, Cy.; Huang, JC. and al. 2008. Novel Technique for preparing anano- silver water suspension by the arc -discharge method. Review on. Advanced materials science, 18: 750 -756.

[7] Trop, M.; Novak, M.; Rodl, S.; LLellbom, B.; Kroell, W. and Goessler, W. 2006. Silver coated dressing Acticoat caused raised liver enzymes and Argyria like symptoms in burn patient. J. Trauma. 60: 648- 655 .

[8] Sardari, R. R. R.; Zarchi, S. R.; Talebi, A.; Wang, S.; Wang, J.; Liu, L.; J.; Li, J.; Yuan, F.; Lu, S.; Wan, Z.; Chou, L. and Xi, T. 2012. Toxicological effects of silver nanoparticles in rats. Afr. J. Microbiol. Res 6(27):5587- 5593.

[9] Attia, A. A. 2014. Evaluation of the testicular alterations induced by silver nanoparticles in male mice: Biochemical, Histological and ultrastructural

\title{
Effect of the Silver Nanoparticles on the Histology of Albino Mice Ovaries
}

\author{
Gazwa D.Al-Nakeeb ${ }^{1}$ \\ Noor Mahdi Fatheel ${ }^{2}$ \\ ${ }^{1,2}$ Department of Biology, College of Science for Women, Baghdad University, Baghdad, Iraq.
}

Received 28/2/2017

Accepted 24/5/2017

\begin{abstract}
:
The aim of study is to identify the histological changes in ovaries of the albino mice exposed to silver nanoparticles. Sixty adult females were collected and exposures by 4000 p.p.m. and 50-150 nm in size, Females were divided into 3treated groups. The concentration dosage was (1, 1.5 and 2) p.p.m. of silver nanoparticles for 7, 14 and 21 days as exposure periods as well as control group which treated by normal saline. Treated groups appeared different histopathological changes, it is depending on the concentration of silver nanoparticles and the period of exposure. These changes were included congestion in the blood vessels, hemorrhage, hyaline degeneration, fatty degeneration, pyknosis, necrosis as well as fusion of cells in follicular cells, amyloid proteins in the blood vessels and Fatty necrosis.
\end{abstract}

Keywords: Silver Nanoparticles, Ovary, Histopathological effects. 